\title{
Rememoração: contribuições para a compreensão do processo de aprendizagem de conceitos científicos
}

\author{
João Roberto Ratis Tenório da Silva \\ Universidade Federal de Pernambuco - Caruaru - PE - Brasil \\ Maria da Conceição Diniz Pereira de Lyra \\ Universidade Federal de Pernambuco - Recife - PE - Brasil
}

\begin{abstract}
Resumo
Desde a Grécia Antiga a memória é compreendida, sobretudo, como um local de armazenamento de informações. Consideramos que tal modelo é limitado para a compreensão do processo de aprendizagem de conceitos científicos. Neste artigo, tivemos como objetivo investigar como se dá o processo de aprendizagem desses conceitos, em sala de aula, a partir da compreensão do processo de rememoração. Assim, propomos um modelo que considera que, quando o sujeito está numa situação de aprendizagem de um conceito científico, ele ressignifica conhecimentos prévios a partir da rememoração, construindo novos modos de pensar próprios do contexto científico. Acreditamos que nosso modelo pode contribuir para compreender o processo de aprendizagem, levando em consideração o processo de rememoração. Estimulamos, assim, estudos empíricos que, além de ajudarem a melhorar o modelo, podem nos mostrar indícios de como funciona o processo de aprendizagem numa visão holística, permitindo propor, futuramente, métodos de ensino.
\end{abstract}

Palavras-chave: Memória; conceito; aprendizagem.

\section{Remembering: contributions to the understanding of the learning process of scientific concepts}

\begin{abstract}
From ancient Greece memory is understood, above all, as a place of information storage. We consider that such a model is limited for understanding the process of learning scientific concepts. In this article, we aimed to investigate how the learning process of these concepts occurs in the classroom, by understanding the recall process. Thus, we propose a model that considers that, when the student is in a situation of learning a scientific concept, he/she reconfigures previous knowledge from the recollection, constructing new modes of thinking proper to the scientific context. We believe that our model can contribute to understanding the learning process, taking into account the recall process. Thus, we stimulate empirical studies that, in addition to helping to improve the model, can show us how the learning process works in a holistic perspective, allowing future teaching methods to be proposed.
\end{abstract}

Keywords: Memory; concept; learning.

\section{Rememoración: contribuciones a la comprensión del proceso de aprendizaje de conceptos científicos}

\section{Resumen}

Desde la Antigua Grecia la memoria es comprendida, especialmente, como un sitio de almacenamiento de informaciones. Consideramos que tal modelo es limitado para la comprensión del proceso de aprendizaje de conceptos científicos. En este artículo, tuvimos como objetivo investigar cómo se da el proceso de aprendizaje de esos conceptos, en sala de clase, a partir de la comprensión del proceso de rememoración. Así, proponemos un modelo que considera que, cuando el sujeto está en situación de aprendizaje de un concepto científico, él resignifica conocimientos previos a partir de la rememoración, construyendo nuevos modos de pensar propios del contexto científico. Creemos que nuestro modelo pueda contribuir para comprender el proceso de aprendizaje, llevando en consideración el proceso de rememoración. Estimulamos, así, estudios empíricos que, además de ayudar a mejorar el modelo, puedan enseñarnos indicios de cómo funciona el proceso de aprendizaje en una visión holística, permitiendo proponer, futuramente, métodos de enseñanza.

Palabras clave: Memoria; concepto; aprendizaje. 


\section{Introdução}

Segundo Wagoner (2011), estudos sobre a memória datam da Grécia Antiga, quando se considerava que tal processo era uma dádiva da deusa Mnemosine, e o contemplado por essa dádiva deveria propagar suas memórias. Além disso, Platão tinha a ideia de que a memória funcionava como um bloco de cera, em que as experiências de vida eram cravadas, o que culminou em metáforas que são utilizadas até hoje, em que a memória é considerada como um local de armazenamento ou de inscrição de informações.

Séculos mais tarde, já no âmbito da Psicologia Experimental, Ebbinghaus (1885/1913) desenvolveu uma abordagem que reforçou a noção de memória como inscrição de informações num substrato material. No experimento com sílabas sem sentido, ele elaborou uma curva de esquecimento a partir de repetições em série que fazia com ele mesmo. Assim, Ebbinghaus(1985/1913) percebeu que quanto mais as sílabas eram repetidas, mais facilmente a sequência era memorizada. Como consequência, ele concluiu que quanto mais repetimos as informações, mais profundamente elas são gravadas em nosso substrato mental, impedindo que sejam esquecidas.

Essas metáforas, que podemos chamar de espaciais (Wagoner, 2011), continuam a ser exploradas em alguns estudos na Psicologia Cognitiva, tais como aqueles apresentados por McGaugh (1966), Cowan (1988), Zola-Morgan e Squire (1990) e Kandel (2001). Porém, contrapondo-se às metáforas sobre a memória como inscrição de informações armazenadas, neste trabalho a consideramos como um processo semiótico que, construindo significados, regula a adaptação do sujeito ao ambiente e, juntamente com outros processos cognitivos e afetivos, tem um papel essencial no desenvolvimento humano e na aprendizagem (Zittoun \& cols., 2011). Esse processo de adaptação funciona envolvendo também a atenção, a percepção e a imaginação, além da afetividade e da inserção do sujeito em um meio social e cultural (Bartlett, 1932; Halbwachs, 1992).

Entendemos que esta noção de memória pode nos oferecer indícios de como funciona o processo de aprendizagem de conceitos científicos. Neste sentido, o foco está na investigação da capacidade do sujeito de usar experiências do passado para projetá-las no futuro, face a tarefas que visam a aprendizagem desses conceitos.

$\mathrm{Na}$ literatura, encontramos perspectivas teóricas, tais como a teoria sociocultural (Vygotsky, 1962) e o construtivismo piagetiano (Piaget,1974), que deram origem a uma série de ideias de como funciona o processo de aprendizagem. Para essas perspectivas, o ambiente surge como um elemento essencial no desenvolvimento humano, sendo a diferença entre elas, a forma pela qual o sujeito irá interagir com o seu contexto. Enquanto que para Piaget os conceitos de assimilação e acomodação se tornam centrais para compreensão da aquisição de novas habilidades e conceitos, com uma ênfase no meio físico, em Vygotsky encontramos na sociogênese a chave para a compreensão de como o sujeito, em seu desenvolvimento, internaliza habilidades e significados que são compartilhados culturalmente (Valsiner \& Van Der Veer, 1988; Van der Veer \& Valsiner, 1988).

Segundo o conceito de internalização, elaborado por Vygotsky (1962), o desenvolvimento cultural do sujeito acontece em dois estágios: primeiramente em um nível social - interpsicológico - e depois em um nível individual - intrapsicológico. Assim, o autor afirma que as funções mentais superiores emergem a partir das trocas sociais sendo, progressivamente, internalizadas. Este processo é mediado pelos signos, sendo a palavra, para Vygotsky, o principal mediador nas relações sociais (Valsiner \& Van Der Veer, 1988; Van Der Veer \& Valsiner,1988). A todo o momento, o sujeito internaliza elementos do seu dia a dia, a partir de sua relação com o ambiente (relações sociais). Porém, a forma como os significados, compartilhados culturalmente, são internalizados, não será a mesma para todas as pessoas. O modo pelo qual o sujeito interage com o ambiente irá determinar como os significados serão internalizados (Vygotsky,1994).

Valsiner (2007/2012), ao elaborar a noção de internalização proposta por Vygotsky afirma que a internalização é o processo de análise da experiência externa de materiais semióticos e sua síntese numa nova forma no domínio intrapsicológico, ou seja, diz respeito a como os sujeitos constroem significados sobre suas experiências no e do mundo. Além disso, Valsiner (2007/2012) destaca o papel da externalização, que se caracteriza como um processo de análise dessas experiências, em nívelsubjetivo (materiais pessoais-culturais) e a sua transposição para o mundo externo, como de "dentro" para "fora" da pessoa. Nessa transposição, a pessoa comunica a sua nova síntese, podendo modificar, dessa forma, o ambiente externo (Valsiner, 2007/2012).

Diante da discussão apresentada até aqui, consideramos que uma análise do processo pelo qual o sujeito externaliza seus conhecimentos prévios (experiências passadas), diante de uma situação de aprendizagem (posta em sala de aula), pode desencadear a construção de novos significados sobre algum conceito científico específico. A externalização se dá a partir do processo de rememoração, em que, segundo Bartlett (1932), Valsiner (2007/2012) e Wagoner (2011), promove a construção de novos significados, visto que novos elementos são agregados ao que está sendo rememorado. Logo, a internalização de conceitos científicos em sala de aula não se dá, puramente, a partir de uma transmissão de significados já estabelecidos dentro de uma comunidade científica, mas a partir de um jogo dialético, estabelecendo tensões e oposições, entre o que está sendo posto em sala de aula (visão do conceito estabilizada em um contexto científico) e os conhecimentos prévios (experiências passadas) que os alunos irão rememorar para compreensão do conceito. É, justamente, o estudo desse processo que destacamos no presente trabalho, no qual temos como objetivo investigar como se dá o processo de aprendizagem de conceitos científicos, em sala de aula, a partir da compreensão do processo de rememoração. Ou seja, como o sujeito recupera e ressignifica o material internalizado anteriormente, ao externalizá-lo face a demandas presentes em situações/problemas de aprendizagem. Ademais, como este 
processo vai adquirindo estabilidade, aproximando a compreensão do conceito científico em estudo.

\section{Aprendizagem como processo semiótico de construção de significados}

Quando olhamos para as ideias de Vygotsky (1934, 1962) sobre aprendizagem, identificamos o surgimento de conceitos que são determinantes na explicação do processo de aprendizagem, voltados para a relação do sujeito com seu meio social e cultural. Além do conceito de internalização, apresentado anteriormente, a noção de signo surge na teoria de Vygostky como central em sua abordagem.

Para Vygotsky $(1962,1978)$, a noção de aprendizagem é construída a partir da consideração de que o mundo é internalizado na interação do sujeito com o mundo social ou o outro social, sendo esta interação mediada por signos (instrumentos psicológicos socialmente originados). Durante esse processo, o sujeito internaliza os produtos de sua cultura, com os significados dos objetos assumidos nas relações sociais. Ao externalizar, o sujeito pode atribuir novo significado (ressignificar) de maneira pessoal (Valsiner, 2009; 2007/2012).

Segundo Valsiner (1998), a existência humana é organizada por significados semióticos - signos de diferentes tipos - socialmente construídos e pessoalmente internalizados. Signos, segundo o autor, são representações de algum aspecto do fenômeno experienciado e são construídos por alguém para suprir as necessidades de comunicação - com outras pessoas e consigo mesmo. Este ato construtivo é sempre pessoal, requerendo um ser humano ativo em relação à sua cultura (cultura pessoal) e à cultura coletiva por meio dos processos de internalização e externalização (Valsiner, 2007/2012).

Valsiner (2009) afirma que as experiências passadas regulam o novo horizonte de aprendizagem do aluno. No processo de aprendizagem, a ação no presente (uso de materiais instrucionais, explicação do professor em sala, debate com colegas etc.) irá abrir possibilidades de trajetórias de aprendizagem no futuro, que irão aparecer como zona de desenvolvimento proximal do sujeito (Vygotsky, 1962; Valsiner \& Van Der Veer, 2014).

Compreendemos que a aprendizagem se dá baseada na experiência prévia do sujeito, a qual terá um papel fundamental na zona de desenvolvimento proximal, abrindo novas potencialidades de aprendizagem a partir da ressignificação dessas experiências. Essas potencialidades são possíveis levando em consideração essas experiências passadas (conhecimento prévio), visto que elas orientam o sujeito para o futuro no momento em que ele interage com o ambiente (seja com outros sujeitos ou com materiais de instrução). A projeção do futuro, dentro do horizonte de potencialidade de aprendizagem, é possível devido à influência da imaginação (Zittoun \& cols., 2011). É importante ressaltar que, no momento da aprendizagem, diversos processos cognitivos importantes estão envolvidos, tais como aten- ção, além da memória, percepção, raciocínio, imaginação etc. Não temos como objetivo discutir a relação entre todos esses processos agindo conjuntamente na aprendizagem. Neste artigo, focamos a memória, particularmente no processo de rememoração, numa perspectiva (re)construtiva, explorando como esse processo desencadeia a construção de novos significados.

\section{Memória como processo de ressignificação}

Dada a natureza semiótica da memória (Vygotsky,1978), o que um sujeito lembra e como o faz depende de sua localização no contexto sociocultural na sua trajetória de vida (Mead, 1934; Zittoun \& cols., 2011) e da construção de significados a signos envolvidos nesse processo. Por exemplo, podemos considerar que um conceito científico, particularmente aqueles que transitam no âmbito das concepções do senso comum, como o de calor, adquire formas de estabilidade que vão depender dos significados que atribuímos a eles em determinadas situações e contextos socioculturais ${ }^{1}$. Dentro da comunidade científica, por exemplo, o conceito de calor adquire uma forma mais estável, sendo aplicado em diversas situações para explicar fenômenos assumindo uma visão científica. Já em contextos do cotidiano, o conceito de calor adquire formas menos estáveis, que vão variar dependendo da experiência individual de cada pessoa com o conceito, o contexto e a situação específica na qual esse conceito será usado. Nessas situações, por exemplo, é possível a um sujeito, com formação específica em Química, tratar o calor relacionado às sensações térmicas, mesmo que tal ideia seja equivocada do ponto de vista científico. Isto ocorre devido à sua experiência em situações do cotidiano em que o calor é sempre associado ao "quente" e a sua ausência é associada ao "frio" (Amaral \& Mortimer, 2001). Esse mesmo sujeito, em um contexto de sala de aula ou laboratório, irá tratar o calor do ponto de vista científico, considerando trocas energéticas e movimentos das partículas constituintes dos materiais. Isso significa que alguns conceitos, considerados como polissêmicos (Mortimer, Amaral, \& El-Hani, 2014), vão apresentar determinados modos de pensar e de falar fora do contexto da ciência, que se configuram como conhecimentos prévios, construídos ao longo da vida do sujeito, antes dele ter contato com a visão científica em sala de aula.

Os modos de pensar, que se configuram como conhecimentos prévios, emergem em sala de aula (Azevedo, 2004; Silva \& Amaral, 2016) influenciando o processo de aprendizagem da visão científica de conceitos. Assim, entendemos que se faz necessária uma reflexão de como esses conhecimentos prévios são externalizados em sala de aula, por meio do processo de rememoração (Bartlett,1932).

1 O foco deste artigo está, sobretudo, nesses conceitos, cujos diversos significados estão dispersos em diferentes contextos socioculturais, se caracterizando como polissêmicos (Mortimer, Amaral, \& El-Hani, 2014). Porém, o processo de aprendizagem através da rememoração, proposto neste artigo, também se aplica a conceitos usados exclusivamente em contextos científicos, como o de orbital ou elétron, ambos da Química. 


\section{Teoria da Rememoração de Bartlett}

Bartlett (1932) abre uma nova forma de se investigar e conceituar a memória, conduzindo uma série de experimentos sobre percepção, imaginação e memória. Isso permitiu que ele criasse uma teoria em que se levasse em consideração todos esses processos conjuntamente. Em seus experimentos sobre memória, ele desenvolveu três métodos: 1) método da descrição; 2) método da reprodução em série e 3) método da reprodução repetida.

Durante a análise dos dados construídos através desses três métodos, Bartlett (1932) identificou algumas características gerais do processo de rememoração, tais como:

- Eliminação de detalhes: os participantes tendiam a rememorar características gerais do objeto, eliminando detalhes, deixando a reprodução mais curta, objetiva e concisa em relação ao objeto mostrado originalmente;

- Transformação: os participantes apresentavam a tendência de trocar nomes ou expressões do objeto rememorado;

- Transferências: era frequente a transferência de características de uma reprodução para outra, havendo uma mistura de detalhes.

Com esses dados gerais e uma minuciosa análise do processo por meio do qual a rememoração ocorria, Bartlett (1932) desenvolveu um programa sistemático de pesquisa em Psicologia Social e Experimental, adotando, sobretudo, a concepção de uma mente ativa, de natureza holística e interdependente do grupo social. Para explicar as características do processo de rememoração apontadas acima, Bartlett (1932) propôs o conceito de esquema, que apresentaremos a seguir.

\section{Esquema: agindo no presente a partir da ressignificação de experiências do passado}

Utilizando a noção de esquema de Head (1920), em que esquemas seriam formados por estruturas que armazenavam os movimentos corporais em série, como imagens mentais, que nos faziam capazes de realizar movimentos, Bartlett (1932) construiu sua teoria sobre a memória, porém, fazendo algumas alterações. Segundo Bartlett (1932), esquema é um conceito holístico, que exprime um processo global e não estruturas estáticas que representam imagens mentais, tal como afirmava Head. Dessa forma, Bartlett define que esquemas são uma organização ativa de experiências do passado, os quais são reformulados quando estamos diante de uma demanda no presente. Portanto, esquemas são sempre dinâmicos, construídos a partir de experiências passadas interagindo com que está posto no presente. Bartlett (1932) aponta que as experiências passadas, ao serem reconstruídas no presente, nos ajudam a agir em determinadas situações inesperadas a partir da re- formulação e atualização dos esquemas. Assim, processos imaginativos e perceptuais entram em ação, permitindo uma transição temporal de forma que experiências passadas sejam reformuladas no "aqui e agora".

Para localizar informações sobre o passado, na reformulação de esquemas, o organismo adquire a capacidade de se voltar sobre seus próprios esquemas, reconstruí-los e atualizá-los (Bartlett, 1932), dando significado à experiência do presente a partir da ressignificação de experiências do passado. Nesse momento, o processo de reformulação de esquemas se torna consciente.

\section{Voltando-se sobre os próprios esquemas: preenchendo lacunas no presente}

Segundo Bartlett (1932), normalmente, há a criação de uma descontinuidade quando tentamos nos lembrar de algo para agir no presente. Buscamos, então, informações do passado e, através da imaginação, preenchemos lacunas durante a rememoração. Esse é um aspecto normal do processo de esquecimento, que faz com que nos voltemos sobre nossos próprios esquemas e os transformemos, de forma a corrigir essa descontinuidade. Assim, um sujeito que se volta sobre seus próprios esquemas para cobrir essa descontinuidade, se encontra em um ambiente dual porque está envolvido no passado (usado para controle da autorreflexão nas ações) e no presente (tempo da ação) (Wagoner, 2013). Dessa forma, um sujeito pode reconstruir algo que aconteceu há um ano, reintegrá-lo e combiná-lo com algo que aconteceu ontem, a fim de resolver algum problema que o desafia no dia a dia (Bartlett, 1932). É dessa forma que às vezes nos lembramos de algo, mas não sabemos se tal evento aconteceu na semana passada ou no mês passado, por exemplo.

\section{Aprendizagem e rememoração}

Acreditamos que a concepção sobre memória como (re)construção e o processo de aprendizagem podem dialogar, conduzindo a uma complementação útil à compreensão do processo de aprendizagem de conceitos científicos. Dessa forma, podemos compreender como o sujeito (re)negocia significados durante o processo de aprendizagem. Para isso, partimos de alguns pressupostos:

Conceitos são dinâmicos e apresentam estabilidade relativa

Consideramos que os conceitos não são estruturas estáticas, mas adquirem formas de estabilidade no desenrolar do processo de aprendizagem, numa espécie de evolução do pensamento conceitual (Mortimer, Amaral, \& El-Hani, 2014). Zittoun e cols. (2011) afirmam que nossas vidas são modeladas por campos de significação. Ainda que não percebamos, nós acreditamos que algumas escolhas são baseadas somente em nossas preferências como, por exemplo, para vestir uma determinada roupa. Porém, não 
reconhecemos que essas experiências estão ligadas aos significados que atribuímos a elas. Da mesma forma, na aprendizagem de conceitos científicos, podemos considerar que o pensamento conceitual se modifica dependendo da situação e contexto. A estabilidade de um conceito vai depender dos significados que atribuímos a ele em determinadas situações. Ou seja, alguns conceitos, aqueles considerados polissêmicos, vão apresentar determinados modos de pensar e de falar, e o uso desses modos dependerá do contexto e situação em que o sujeito estará inserido (Mortimer, Amaral, \& El-Hani, 2014). Assim, a aprendizagem se torna um processo de regulação do conceito, em que novos modos de pensar emergem a partir da evolução do conceito como ato do pensamento. Dessa forma, o conceito é ressignificado (novos modos de pensar emergem na relação sujeito-ambiente) dependendo do contexto, da situação em que sua demanda é necessária e da forma pela qual o sujeito interage com o ambiente (Vygotsky, 1934).

Retomando o exemplo do conceito de calor, o aluno usa este conceito em seu dia a dia, o relacionando com a sensação térmica "quente". Porém, no momento de aprendizagem, tal modo de pensar, de conceituar, se desenvolve, pela ressignificação, fazendo com que ele compreenda que essa ideia tem sua delimitação no contexto da ciência, e o calor deverá ser tratado a partir do ponto de vista científico (químico ou físico). Este último ponto de vista é um modo de pensar em que o conceito adquire uma estabilidade maior, quando ele ganha o status de científico. Não podemos afirmar que o conceito de calor, tomado aqui como exemplo, pertence à comunidade científica apenas, sendo um conceito científico, mas seus modos de pensar estão dispersos em diversos contextos sociais e culturais ${ }^{2}$ e a ressignificação desses modos de pensar, num processo de evolução conceitual, auxilia na construção da visão científica do conceito. Em sala de aula, o objetivo se torna o aluno construir modos de pensar sobre o conceito, dentro de uma visão científica, relacionando-o com outros modos de pensar utilizados em outros contextos e, assim, tomar consciência dos diversos modos de pensar que um conceito pode apresentar. Nesse processo, a rememoração auxilia na construção de significados, visto que, como discutido anteriormente, ao rememorar experiências passadas, no nosso caso, conhecimentos prévios, novos significados são construídos, a partir da agregação de diversos elementos ao produto da rememoração.

\section{Rememoração como mecanismo de ressignificação dos conceitos}

Como discutimos anteriormente, no momento em que estamos tentando nos lembrar algo, voltamos aos nossos próprios esquemas (Bartlett, 1932) para cobrir lacunas de memória. Nesse processo, ressignificamos nossas experiências de forma a resolver determinadas demandas do pre-

2 Outros exemplos de conceitos polissêmicos que apresentam diversos significados aplicáveis em diferentes contextos, não só científico, são: espontaneidade/entropia (Amaral \& Mortimer, 2004), substância (Silva \& Amaral, 2013) e energia (Simões Neto \& Amaral, 2016). sente. Ao nos recordarmos de algo, estamos externalizando significados já socialmente constituídos de uma forma diferente de quando foram internalizados. Assim, ao rememorar o conhecimento prévio relativo a qualquer conceito na sala de aula, o sujeito poderá elaborar novos significados a partir da necessidade de atendimento às demandas requeridas em alguma situação de aprendizagem (resolução de problemas, explicação de determinados fenômenos, exposição de seminários, etc.). Tal ressignificação, entendida como uma evolução do conceito no ato do pensamento, é proporcionada pela reformulação de esquemas (Bartlett, 1932), pois uma série de situações de uso de um conceito no contexto científico se combinará com seu uso no cotidiano, para a resolução de uma demanda presente. Para compreender, por exemplo, que o fornecimento de energia na forma de calor é responsável pela dilatação térmica de metais, o sujeito pode rememorar diversas situações em seu dia a dia, como a fervura da água para cozimento de alimentos ou o derretimento do gelo em temperatura ambiente, depois ele pode combinar com outros aspectos conceituais estudados em sala de aula (como a expansão térmica de gases). A partir disso, ele pode construir novos significados (numa visão científica) sobre a expansão térmica dos metais, a qual está relacionada com o aumento da energia cinética das partículas durante o fornecimento de energia (assim como acontece na fervura da água e na expansão dos gases, rememorados anteriormente).

Com base nesses dois princípios, acreditamos que a memória, como processo de rememoração, pode nos dar indícios de como o sujeito estabelece relações entre conhecimentos prévios e os científicos, essencial num processo de aprendizagem, na perspectiva dialética (Vygotsky, 1962). Essas relações irão depender dos significados atribuídos ao conceito, numa relação dinâmica com o ambiente (Vygotsky, 1934; Zittoun \& cols., 2011). A cada nova demanda em um novo contexto, o conceito sofrerá mais ressignificações (ampliações de modos de pensar/construção de novos significados), a partir das constantes reformulações de esquemas de rememoração, durante os processos de internalização e extenalização. Assim, podemos resumir nossa proposta com o diagrama apresentado na Figura 1.

Na Figura 1 ao lado esquerdo, temos um sujeito que apresenta um conhecimento prévio, construído ao longo de sua vida, que o tempo todo, é ressignificado (ampliação de modos de pensar - construção de novos significados), a partir da relação do sujeito com seu ambiente, no uso de instrumentos culturais - signos - e interações sociais. Assim, compreendemos a aprendizagem de conceitos científicos como um processo de ressignificação, em que novos significados, numa visão científica, são construídos e se agregam àqueles já existentes, ampliando os modos de pensar do conceito para o sujeito (Mortimer, Amaral, \& El-Hani, 2014). Porém, a dinâmica que descreve esse processo não se caracteriza por um acréscimo linear desses novos significados, mas envolve tensões e oposições que conduzem a sínteses inovadoras caracterizando, assim, uma dinâmica dialética (Boesch, 2008; Valsiner, 2009, 2007/2012; Valério \& Lyra, 2016), quando o sujeito estabelece relações entre o conhe- 


\section{Rememoração de conhecimentos prévios relevantes na aprendizagem do conceito que levará a mais ressignificações}

Conhecimento prévio

Conhecimento

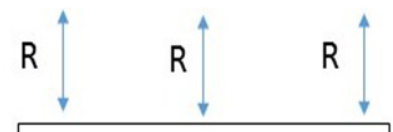

prévio

Conhecimento

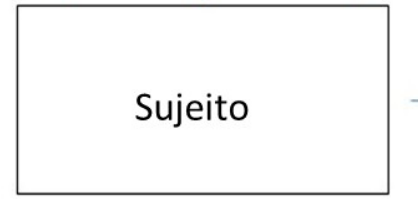

Situações de aprendizagem em sala de aula

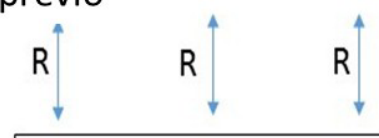
Aprendizagem

Sujeito

\section{$R$ - Ressignificação (constante uso dos conceitos em diversos contextos - ampliação de modos de pensar) $R=$ Internalização + Externalização}

Figura 1. Processo de aprendizagem baseado na rememoração.

cimento prévio e científico, para uso do conceito em novas situações dentro da sala de aula.

Assim, consideramos que a memória, na perspectiva (re)construtiva é um elemento essencial para compreensão do processo de aprendizagem, visto que é através dela que novos significados podem ser construídos. Ressaltamos que todo esse processo que possui essa plasticidade e, ao mesmo tempo, atinge a concepção científica do conceito, ocorre a partir de esquemas nmemônicos reformulados, diante de tensões e oposições de significados concomitantes. Essas são postas ao sujeito pelas demandas presentes e interagem dinamicamente com as experiências passadas. Ademais, as mesmas estão perenemente inseridas no contexto social e cultural do sujeito (Boesch, 2008; Valsiner, 2009, 2007/2012; Valério \& Lyra, 2016).

\section{Considerações Finais}

O objetivo do presente texto foi o de destacar o papel da memória na aprendizagem de conceitos científicos, exemplificados, particularmente, em relação a conceitos que partilham concepções advindas do senso comum, na área de ensino de Química. O ponto central está na compreensão da memória como processo de adaptação, que permite a (re)construção de significados a partir das demandas do presente que visam imaginar possibilidades futuras ainda não vividas. A rememoração concebida como esse processo se fundamenta nas ideias de Bartlett (1932), que a considerava como um processo holístico que envolve outros processos cognitivos e também afetivos, incluindo atenção, percepção, raciocínio, imaginação e uma inserção perene no contexto social e cultural do sujeito.

Possibilidades de investigação dessa temática - rememoração e aprendizagem de conceitos científicos - envolvem o acompanhamento do desenrolar da aprendizagem, procurando identificar mediadores socioculturais que são utilizados pelos sujeitos no decorrer do processo de aprendizagem de conceitos científicos, com base na exploração (re) construtiva da memória. Relevância fundamental é dada à relação desse processo com elementos do meio social e cultural e com as características das tarefas envolvidas em sala de aula. Nesse sentido, entendemos que novas perspectivas de pesquisa estão abertas para que se possa construir um conhecimento que nos dê subsídios para compreender a aprendizagem de conceitos científicos, nos permitindo criar novas dinâmicas na sala de aula de ciências.

A perspectiva aqui assumida adota a ideia de que a construção de significados se dá ao internalizar signos histórica e culturalmente constituídos, mas que são reconstruídos pelos indivíduos numa dinâmica constante através de um processo interdependente de internalização/externalização. Assim, tanto nos filiamos às ideias de Vygotsky $(1934,1978)$ como aos desdobramentos e reconstruções desenvolvidas por Valsiner (2007/2012) e autores como Zittoun e cols. (2011) e Wagoner (2012), que trabalham, mais especificamente, sobre a rememoração, imaginação e aprendizagem. E também Mortimer, Amaral, e El-Hani (2014), os quais se preocupam com a aprendizagem de conceitos científicos em sala de aula.

O modelo proposto neste artigo focaliza o papel da memória reconstrutiva na aprendizagem de conceitos científicos, enfatizando como o conhecimento prévio se transfor- 
ma durante a rememoração no processo de aprendizagem. Nesse processo, o conhecimento prévio do sujeito é ressignificado a partir da reformulação de esquemas (Bartlett, 1932), permitindo a construção de novos significados que serão aplicados em outros contextos.

Consideramos que se fazem necessárias pesquisas para que dados empíricos contribuam para a compreensão do processo de aprendizagem, levando em consideração a memória como mecanismo de reconstrução de significados, ao lado, sobretudo, da imaginação (Zittoun \& cols., 2009). Acreditamos que pesquisas empíricas, utilizando o modelo do processo de aprendizagem aqui proposto, podem nos mostrar indícios de como funciona esse processo adotando uma perspectiva não segmentada, mas sim holística, nele integrando a imaginação.

\section{Referências}

Amaral, E.M.R. \& Mortimer, E.F (2001). Uma proposta de perfil conceitual para o conceito de calor. Revista Brasileira de Pesquisa em Educação em Ciências, 1(3), 1-16.

Amaral, E.M.R. \& Mortimer, E. F. (2004). Un perfil conceptual para entropia y espontaneidad: una caracterizacion de las formas de pensar y hablar em el aula de química. Educacion química, (3),60 - 75.

Bartlett, F.C. (1932). Remembering: a study in experimental and social psychology. New York: Cambridge Univ. Press

Boesch, E.E. (2008). On subjective culture. Culture \& Psychology, 14(4), 498-512.

Azevedo, M. C. P. S. (2004). Ensino por Investigação: problematizando as atividades em sala de aula. Em A. M. P. Carvalho, (Org.), Ensino de ciências: unindo a pesquisa e a prática. São Paulo: Pioneira Thomson Learning.

Cowan, N. (1988). Evolving conceptions of memory storage, selective attention, and their mutual constraints within the human informationprocessing system. Psychological Bulletin, 104(2), 163-191.

Ebbinghaus, H. (1885/1913). Memory: A contribution to experimental psychology. New York: Dover

Halbwachs, M. (1992). On Collective Memory. Chicago: Chicago University Press.

Head, H. (1920). Studies in Neurology (Vol. 2). London: Oxford University Press

Kandel, E. (2001). The Molecular Biology of Memory Storage: A Dialogue Between Genes and Synapses. Science, 294(2),10301038.

McGaugh, J.L. (1966). Time-Dependent Processes in Memory Storage. Science, 153, 1351-1358.
Mortimer, E.F., Amaral, E.M.R., \& El-Hani, C.N. (2014). Conceptual Profiles: Theoretical- Methodological Bases of a Research Program. Em: E.F. Mortimer \& C.N. El-Hani, (Orgs.), A Theory of Teaching and Learning Scientific Concepts Series: Contemporary Trends and Issues in Science Education. Hollanda: Springer. 42, XVII, $330 \mathrm{p}$.

Mead, G.H. (1934). Mind, Self and Society: From the standpoint of a social behaviorist. Chicago: Chicago University Press.

Piaget, J. (1974). A Epistemologia Genética e a Pesquisa Psicológica. Rio de Janeiro: Freitas Bastos

Silva, J.R.R.T. \& Amaral, E.M.R. (2013). Proposta de um Perfil Conceitual para Substância. Revista Brasileira de Pesquisa em Educação em Ciências, 13(3).

Simões Neto, J.E. \& Amaral, E.M.R. (2016). Trajetória Histórica para a Compreensão da Energia e Concepções de Licenciandos de Química e Física sobre esse conceito. Anais do XVIII Encontro Nacional de Ensino de Química (XVIII ENEQ). Florianópolis, SC, Brasil: UFSC.

Valério, T.A. \& Lyra, M.C.D.P. (2016). Significados ambivalentes no processo de adoção: um estudo de caso. Psicologia em Estudo, 12, 211-222.

Valsiner, J. (2009) Constructing the vanish present between the future and the past. Infancia y Aprendizaje, 34(2), 141-150

Valsiner, J. (2012). Fundamentos da Psicologia Cultural - Mundos da Mente Mundos da Vida. Porto Alegre: Artmed. (Trabalho original publicado em 2007).

Valsiner, J. \& Van der Veer, R. (1988): On the social nature of human cognition. Journal for the Theory of the Behavioral Sciences, 18, 11735

Valsiner, J. \& Van der Veer, R. (2014). Encountering the Border: Vygotsky's zona blizaishegorazvitia and its implications for theories of development. Em A. Yasnitsky \& R. Van der Veer (Orgs.), Handbook of Cultural-Historical Psychology. Cambridge: Cambridge University Press

Van der Veer, R. \& Valsiner, J. (1988). Lev Vygotsky and Pierre Janet. On the origin of the concept of sociogenesis. Developmental Review, 8, 5265.

Vygotsky, L.S. (1962). Thought and Language. Cambridge: MIT Press.

Vygotsky, L.S. (1978). A formação social da mente (2a.ed., J.C. Neto, L.S.M. Barreto, \& S.C.A., Trad.). São Paulo: Martins Fontes.

Vygotsky, L. S. (1994). The problem of the environment. Em R. Van der Veer \& J. Valsiner (Orgs.), The Vygotsky Reader (pp. 338-354). Oxford: Blackwell Publishers. 
Wagoner, B. (2011). Meaning construction in remembering: Asynthesis of Bartlett and Vygotsky. Em P. Stenner, J. Cromby, J. Motzkau, \& J. Yen (Orgs.), Theoretical Psychology: Global Transformations and Challenges (pp. 105-114). Toronto: Captus Press

Wagoner, B. (2012) Culture in Constructive Remembering. Oxford handbook of culture and psychology.
Zittoun, T., Valsiner, J., Vedeler, D., Salgado, J., Gonçalves, M., \& Ferring, D. (2011). Melodies of living: Developmental science of human life course. Cambridge: Cambridge University Press

Zola-Morgan, S. \& Squire, L. R. (1990). The primate hippocampal formation: Evidence for a time limited memory storage. Science, 250, 288-290.

Recebido em: 05/11/2015

Reformulado em: 10/09/2016

Aprovado em: 17/10/2016

\section{Sobre os autores}

João Roberto Ratis Tenório da Silva (joaoratistenorio@gmail.com)

Programa de Pós-graduação em Psicologia Cognitiva. Universidade Federal de Pernambuco.

Maria da Conceição Diniz Pereira de Lyra (marialyra2007@gmail.com)

Doutora em Psicologia. Universidade Federal de Pernambuco.

Este artigo é fruto de discussões teóricas acerca do tema de tese de doutorado do autor principal. Neste trabalho são apresentadas elaborações teóricas sobre o processo de rememoração, visando a proposição de um modelo da aprendizagem de conceitos científicos, como será proposto na tese. Não há conflitos de interesse e nem houve recebimento de auxílio financeiro para o desenvolvimento deste trabalho. 